A\&A 381, 757-760 (2002)

DOI: $10.1051 / 0004-6361: 20011572$

(c) ESO 2002

\title{
A new list of extra-galactic radio jets ${ }^{\star}$
}

\author{
F. K. Liu ${ }^{1,2}$ and Y. H. Zhang ${ }^{2}$ \\ 1 Department of Astronomy and Astrophysics, Göteborg University \& Chalmers University of Technology, \\ 41296 Göteborg, Sweden \\ 2 International School for Advanced Studies, Via Beirut 2-4, 34014 Trieste, Italy
}

Received 19 June 2001 / Accepted 11 October 2001

\begin{abstract}
A catalogue of extra-galactic jets is very useful both in observational and theoretical studies of active galaxies. With the use of new powerful radio instruments, the detailed structures of very compact or weak radio sources are investigated observationally and many new radio jets are detected. In this paper, we give a list of 661 radio sources with detected radio jets known to us prior to the end of December 2000. All references are collected for the observations of jets in radio, IR, optical, UV and X-ray wave-bands.
\end{abstract}

Key words. galaxies: general - galaxies: active - galaxies: jets - BL Lac objects: general - quasars: general galaxies: Seyfert

\section{Introduction}

Theoretically every extra-galactic radio source contains at its center an accretion disc surrounding a super-massive black hole and ejecting two symmetric relativistic motion plasma jets in opposite directions along its rotation axes (Begelman et al. 1984). Whether a jet can be detected depends not only on the properties of the radio source itself but also on the instruments used. Radio jets have been detected in various types of radio sources: FR I and FR II radio galaxies, Seyfert galaxies, radio quasars, and BL Lac objects (Bridle \& Perley 1984; Liu \& Xie 1992 hereafter Paper I), though the detected rates of jets are different in different categories of radio sources with the same instruments under similar working conditions. The different detected rates of jets can be reconciled with the unification model of active galaxy nuclei (AGNs) (Bridle \& Perley 1984; Antonucci 1993). By studying jets, one can learn the physics of a jet itself, the ambient medium, and the central engine of the radio source. Many observational works have been dedicated to the studies of the structures of galaxies (e.g. Leahy et al. 1997 and references therein; Owen \& Ledlow 1997 and references therein; Xu et al. 1993 and references therein; Morganti et al. 1993; Condon et al. 1991; Parma et al. 1991; Carilli et al. 1997), radio

Send offprint requests to: F. K. Liu, e-mail: fkliu@fy.chalmers.se

* Table 1 and references to Table 1 are only available in electronic form at the CDS via anonymous ftp to cdsarc.u-strasbg.fr $(130.79 .128 .5)$ or via http://cdsweb.u-strasbg.fr/cgi-bin/qcat?J/A+A/381/757 quasars and BL Lac objects (Lonsdale et al. 1993; Murphy et al. 1993; Bridle et al. 1994; Price et al. 1993; Akujor et al. 1994; Garrington et al. 1991; Kollgaard et al. 1992; Laurent-Muehleisen et al. 1993) since the publication of the jet list given by Liu \& Xie (1992). These works describe a large number of kpc-scale jets in many radio sources. With Very Large Baseline Array (VLBA) and global Very Long Baseline Interferometer (VLBI), the structures of the center of many radio sources are investigated in detail and pc-scale jets (e.g. Spencer et al. 1991; Taylor et al. 1994 and references therein; Dallacasa et al. 1995; Fey \& Charlot 1997 and references therein) are revealed in many compact steep spectrum radio sources and flat-spectrum radio sources. A recent review is given by Zensus (1997) on the understanding of the properties of pc-scale radio jets in extra-galactic radio sources.

In their paper, Bridle \& Perley (1984) list 125 confirmed and 73 possible extra-galactic radio jets. In 1992, Paper I gave 276 radio sources with detected jets known to them prior to mid-December 1989. As many more detected extra-galactic radio jets have been reported in the literature since then, it is time to give an updated list of radio jets. To make the new list in this paper consistent with those reported in the literature, we adopted the morphological definition of a radio jet given by Bridle and Perley (1984). In addition to the information on luminosities of jets, total sources and central cores, the length, sidedness and simple code of morphology were also given in this work. A detailed description of the catalogue will be given in Sect. 2. A simple discussion and conclusion will be given in Sect. 3. Jet properties in IR, optical, UV, 
and X-ray bands will be discussed later (Liu et al. 2001). A Friedmann standard cosmology model is adopted and Hubble constant $H_{0}=100 \mathrm{~km} \mathrm{~s}^{-1} \mathrm{Mpc}^{-1}$ and deceleration factor $q_{0}=0.5$ are used throughout this paper.

\section{Description of the catalogue of known radio jets}

To distinguish a jet from other narrow features or bridges, we used, as in Paper I, the criteria given by Bridle \& Perley (1984) and Bridle (1986) as has been adopted often in the literature: a "jet" is a narrow feature that must be

(a) at least four times as long as it is wide,

(b) separable at high resolution from other extended structures (if any) either by brightness contrast or spatially, and

(c) aligned with the radio nucleus of the parent object where it is closest to it.

Under this definition of a jet, some sources reported to have detected jets, e.g. some core-jet sources observed with VLBI, are not included in our catalogue.

The term "core" used in Bridle \& Perley (1984) and Paper I is resolution-dependent. Bridle et al. (1994) define a "resolution-independent" term "central feature" as an unresolved feature coinciding to within observational errors with the best available position for optical counterparts. It is not always true that a resolution high enough to resolve an opaque feature can be reached in the observations and therefore a "central feature" is still resolutiondependent. Therefore, we still used the term "core" as in Paper I. The usual diagnostics for identification of the nucleus is the positional coincidence with the optical object and flat or inverse radio spectra. Additional constraints are often found from evidence of variability and polarization characteristics. To resolve as clearly as possible the extended emission from the core, we use observational data at a high frequency.

Table 1 lists 661 radio sources with detected radio jets known to us prior to the end of December 2000. All jets in the table were collected from the literature available to us, based on the above definitions. In Table 1, Col. 1 gives the IAU name of the radio source in order of right ascension at 1950.0 and the jet position angle at the nearest to the core - the letters N, E, S, W, NE, SE, NW, and SW represent north, east, south, west, north-east, south-east, north-west, and south-west, respectively. We used the position angle to identify the jets in a radio source. If the difference of position angles of a jet on pc- and kpc- scales is significant, we used the one detected on the kpc-scale. If the jet is one-sided (for the definition of sidedness, see the explanation of Col. 8) or one jet is significantly brighter than the other one in two-sided sources, the letters C-J follow the counter (weaker) jet. If two or more radio galaxies with detected jets are found in a cluster with one IAU designation, we use letters A and B to label them according to their right ascension. Many radio sources are well-known in the literature by their alias name. Column 2 lists some of those names.
Column 3 gives the optical identification of a source - Seyfert galaxies (S), radio galaxies $(\mathrm{G})$ other than Seyferts, radio quasars $(\mathrm{Q})$, or BL Lac object (BL) and its red-shift. A red-shift given in brackets is uncertain. Fifty-three radio sources have no identification in the table. We identify Seyfert galaxies, based on the catalogue of quasars and active nuclei given by Véron-Cetty \& Véron (1996). The numbers 1, 2, and 3 imply type 1, 2, and 3 Seyferts. Identification and red-shift can usually be found in the literature reference given in the Col. 10 or the references therein. However, some sources have no identification and measured red-shift when the radio observations are done. We find the information of the identification and red-shift from the following papers or catalogues: Hewitt \& Burbidge (1993), Junkkarinnen et al. (1991), Véron-Cetty \& Véron (1996), de Vaucouleurs et al. (1991), Sandage \& Tammann (1987). The red-shift of $1826+796$ is taken from Henstock et al. (1997). The radio source 3C 22 was classified as a radio galaxy in the original survey and is re-classified as a quasar by Rawlings et al. (1995).

Columns 4, 5 and 6 list, in the observer's frame, the jet, total and core powers $-\lg P_{\mathrm{j}}^{1.4}$ at $1.4 \mathrm{GHz}, \lg P_{\mathrm{t}}^{1.4}$ at $1.4 \mathrm{GHz}$ and $\lg P_{\mathrm{c}}^{5}$ at $5 \mathrm{GHz}$, respectively. We used the values at these frequencies because the flux densities $S_{\mathrm{j}}^{1.4}$, $S_{\mathrm{t}}^{1.4}$, and $S_{\mathrm{c}}^{5}$ values are most often available. Images at $5 \mathrm{GHz}$ have higher resolution than at $1.4 \mathrm{GHz}$ and thus give flux of a core with less contribution from extended structures. However, the images at $1.4 \mathrm{GHz}$ give higher sensitivity and less observational missing of radio flux. We try to use integrated flux densities for jets with a correction to background (see Bridle et al. 1994 for detail), if possible. When the total flux density of a source is not given in the observational papers of jets or the references therein, we took it from the catalogue by Véron-Cetty \& Véron (1996). We estimated core flux, if not available. We use "typical" values for variable total and core emission. Some $S_{\mathrm{j}}^{1.4}, S_{\mathrm{t}}^{1.4}$, and $S_{\mathrm{c}}^{5}$ values are estimated with extrapolation or interpolation from neighboring frequencies, assuming power-law spectra with a spectrum index of $\nu^{-0.65}$ for the jets, $\nu^{-0.7}$ for the total emission and $\nu^{0}$ for the cores if no spectrum information is available.

In Col. 7 is given the projected length of each jet, $d_{\mathrm{j}}$, in kilo-parsecs, measured from the core (nucleus) over all the jet region.

Column 8 classifies the sidedness of the outer $90 \%$ of the jets. We adopted the same definition as in Bridle (1986) and Bridle \& Perley (1984): one side "1", two side " 2 ", and transition "T", according to the ratio of luminosities between the brighter and fainter jets, larger than 4 , less than 4 , or intermediate. The ratio is usually higher near the core and becomes smaller towards the end of a jet. There is no special physical reason to use 4 instead of other values.

Codification of the morphology of a jet is given in Col. 9 - C-symmetry (C), S-symmetry (S), L-shape (L), very complicated (B) and almost straight (blank). Jet morphology provides information about a jet, interaction 
Table 2. Statistics of Table 1.

\begin{tabular}{|c|c|c|c|c|c|c|c|c|c|}
\hline \multirow{2}{*}{ Type } & \multirow{2}{*}{ Number } & \multicolumn{2}{|c|}{$Z$} & \multicolumn{2}{|c|}{$\lg P_{\mathrm{j}}^{1.4}$} & \multicolumn{2}{|c|}{$\lg P_{\mathrm{t}}^{1.4}$} & \multicolumn{2}{|c|}{$\lg P_{\mathrm{c}}^{1.4}$} \\
\hline & & Min & Max & Min & Max & Min & Max & Min & Max \\
\hline QSOs & 243 & 0.104 & 3.886 & 24.74 & 27.94 & 25.34 & 28.76 & 23.67 & 28.48 \\
\hline G & 279 & 0.0012 & 3.395 & 20.99 & 27.84 & 21.68 & 27.94 & 20.38 & 27.09 \\
\hline $\mathrm{S}$ & 50 & 0.002 & 0.518 & 20.54 & 25.42 & 21.34 & 27.92 & 19.28 & 25.54 \\
\hline BL & 36 & 0.0177 & 1.404 & 22.15 & 27.44 & 23.68 & 28.15 & 23.41 & 28.20 \\
\hline U & 53 & - & - & - & - & - & - & - & - \\
\hline
\end{tabular}

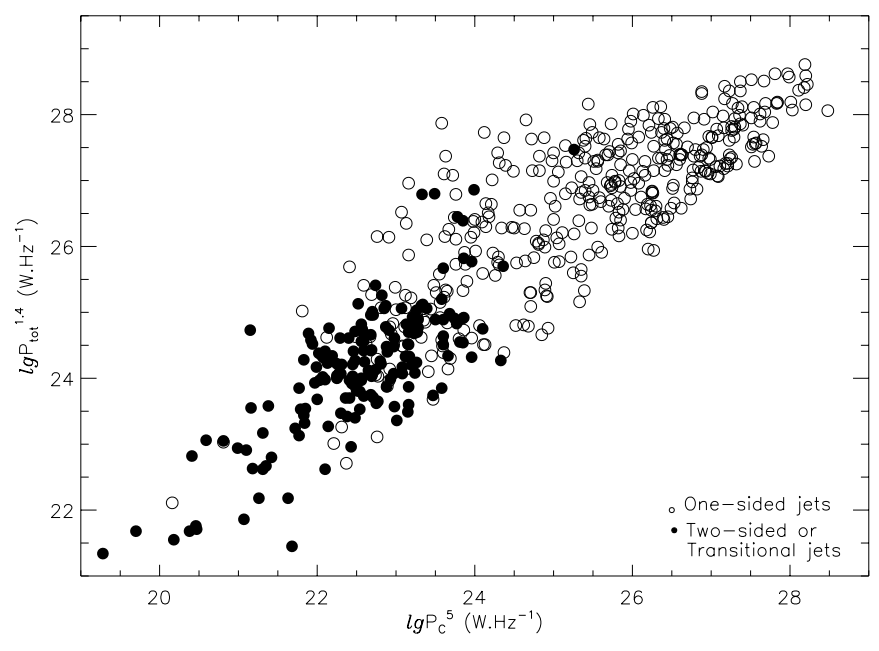

Fig. 1. Change of the sidedness of jets with source and core powers.

between a jet and the intergalactic medium (IGM), the central engine and its motion in the IGM. Narrow-angle tail sources usually have jets of $\mathrm{C}$ shape.

Original references reporting the detection of a jet in radio, infrared, optical, ultraviolet, and X-ray wave-bands are given in Col. 10. Bridle \& Perley (1984) is always given as one of the references, if the source is listed in their catalogue.

The general statistics of Table 1 is given in Table 2 . Column 1 gives the optical type of radio sources. The unidentified objects are listed as U. Column 2 gives the source number. Columns 3, 5, 7 and 9 are the minima and 4, 6, 8 and 10 the maxima of red-shift, total, jet, and core powers at $1.4 \mathrm{GHz}, 1.4 \mathrm{GHz}$ and $5 \mathrm{GHz}$, respectively. The trend of the sidedness of jets varying with the core and total powers is given in Fig. 1. All BL Lac objects, radio quasars and high luminosity radio galaxies are one-sided.

\section{Discussion}

Jets are detected in the red-shift range from $Z_{\min }=0.0012$ to $Z_{\max }=3.886$. The closest radio source with detected jets is the nearest active galaxy Cen A $(1327+427)$ at $3.7 \mathrm{Mpc}$. Jets are detected in QSOs in a red-shift range from $Z_{\text {min }}=0.104$ to $Z_{\max }=3.886$. The minimum value is very close the lower limit of red-shift defining a QSO in Hewitt \& Burbidge (1993). The maximum of the redshift dramatically increases from 2.877 in Paper I to 3.886 . Because of the importance of high red-shift radio galaxies in the study of the formation and evolution of galaxies, many very powerful instruments have been dedicated to the observations of radio galaxies with high redshift and consequently brought about the detection of a large number of jets. The highest red-shift for radio galaxies in Paper $\mathrm{I}$ is 0.574 , which is much smaller than 3.395 given in Table 2. The ranges of total, jet, and core powers do not change a lot, and are comparable to those in Paper I.

Observations of compact steep-spectrum sources is another important subject in the theory of galaxy formation. Only pc-scale jets are detected in compact flat-spectrum and steep-spectrum sources. In the galaxy formation theory, compact steep-spectrum sources are at the early stages of the evolution of AGNs and the detected pc-scale jets are instrinsically small. On the other hand, compact flat-spectrum sources are active galaxies with a small view angle of the jet to the line of sight and the pc jets are the projections of the kpc jets.

We classify radio sources as radio galaxies, radio quasars, BL Lac objects, and Seyfert galaxies, based on the optical properties of the sources. Jets are commonly detected in all types of sources with different detection rates. Large-scale jets are detected in a large fractions of weak radio galaxies and extended radio quasars, although the fraction becomes lower in the lower range of power of core radio emission (Parma et al. 1991). This may result from the selection effect as a consequence of the correlation between opening angle and radio power (Bridle 1986; Parma et al. 1991; Paper I). Jets are detected in a smaller fraction of distant radio galaxies than in radio quasars with similar radio power and with instruments operating under similar parameters.

We gave in this paper a new list of 661 extra-galactic radio sources with detected jets. The list has two significant features: pc-scale jets are detected in many compact flat-spectrum and compact steep-spectrum sources and kpc-scale jets are detected in a large number of radio galaxies and radio quasars.

Acknowledgements. We are greatly indebated to Dr. A. Lanza for his helpful discussion. We thank the referee Dr. R. Perley for his useful comments. FKL acknowledges support by the Swedish Natural Science Research Council (NFR). He also thanks the director and staff of the Department of Astronomy 
and Astrophysics, Göteborg University \& Chalmers University of Technology, for their warm hospitality.

\section{References}

Akujor, C. E., Lüde, E., Browne, I. W. A., et al. 1994, A\&AS, 105,247

Antonucci, R. 1993, ARA\&A, 31, 473

Begelman, M. C., Blandford, R., \& Rees, M. J. 1984, Rev. Mod. Phys., 56, 255

Bridle, A. H. 1986, Can. J. Phys., 64, 353

Bridle, A. H., Hough, D. H., Lonsdale, C. J., Burns, J. O., \& Laing, R. A. 1994, AJ, 108, 766

Bridle, A. H., \& Perley, R. A. 1984, ARA\&A, 22, 319

Carilli, C. L., Röttgering, H. J. A., van Ojik, R., Miley, G. K., \& van Breugel, W. J. M. 1997, ApJS, 109, 1

Condon, J. J., Frayer, D. T., \& Broderick, J. J. 1991, AJ, 101, 362

Dallacasa, D., Fanti, C., Fanti, R., Schilizzi, R. T., \& Spencer, R. E. 1995, A\&A, 295, 27

de Vaucouleurs, G., de Vaucouleurs, A., Jr. Corwin, H. G., et al. 1991, Third Reference Catalogue of Bright Galaxies (Springer-Verlag, New York)

Fey, A. L., \& Charlot, P. 1997, ApJS, 111, 95

Garrington, S. T., Conway, R. G., \& Leahy, J. P. 1991, MNRAS, 250, 171

Henstock, D. R., Browne, I. W. A., Wilkinson, P. N., \& McMahon, R. G. 1997, MNRAS, 290, 380

Hewitt, V., \& Burbidge, G. 1993, ApJS, 87, 451

Junkkarinnen, V., Hewitt, A., \& Burbidge, G. 1991, ApJS, 77, 203
Kollgaard, R. I., Wardle, J. F. C., Roberts, D. H., \& Gabuzda, D. C. 1992, AJ, 104, 1687

Laurent-Muehleisen, S. A., Kollgaard, R. I., Moellenbrock, G. A., \& Feigelson, E. D. 1993, AJ, 106, 875

Leahy, J. P., Black, A. R. S., Dennett-Thorpe, J., et al. 1997, MNRAS, 291, 20

Liu, F. K. 2001, preparation

Liu, F. K., \& Xie, G. Z. 1992, A\&AS, 95, 249 (Paper I)

Lonsdale, C. J., Barthel, P. D., \& Miley, G. K. 1993, ApJS, 87, 63

Morganti, R., Kileen, N. E. B., \& Tadhunter, C. N. 1993, MNRAS, 263, 1023

Murphy, D. W., Browne, I. W. A., \& Perley, R. A. 1993, MNRAS, 264, 298

Owen, F. N., \& Ledlow, M. J. 1997, ApJS, 108, 41

Parma, P., Cameron, R. A., \& de Ruiter, H. R. 1991, AJ, 102, 1960

Price, R., Gower, A. C., Hutchings, J. B., et al. 1993, ApJS, 86,365

Rawlings, S., Lacy, M., Sivia, D. S., \& Eales, S. A. 1995, MNRAS, 274, 428

Sandage, A., \& Tammann, G. A. 1987, A Revised ShapleyAmes Catalog of Bright Galaxies

Spencer, R. E., Schilizzi, R. T., Fanti, C., et al. 1991, MNRAS, 250, 225

Taylor, G. B., Barton, E. J., \& Ge, J. P. 1994, AJ, 107, 1942

Véron-Cetty, M.-P., \& Véron, P. 1996, ESO Sci Rep., No. 17

Xu, W., Readhead, A. C. S., Pearson, T. J., Polatidis, A. G., \& Wilkinson, P. N. 1995, ApJS, 99, 297

Zensus, J. A. 1997, ARA\&A, 35, 607 